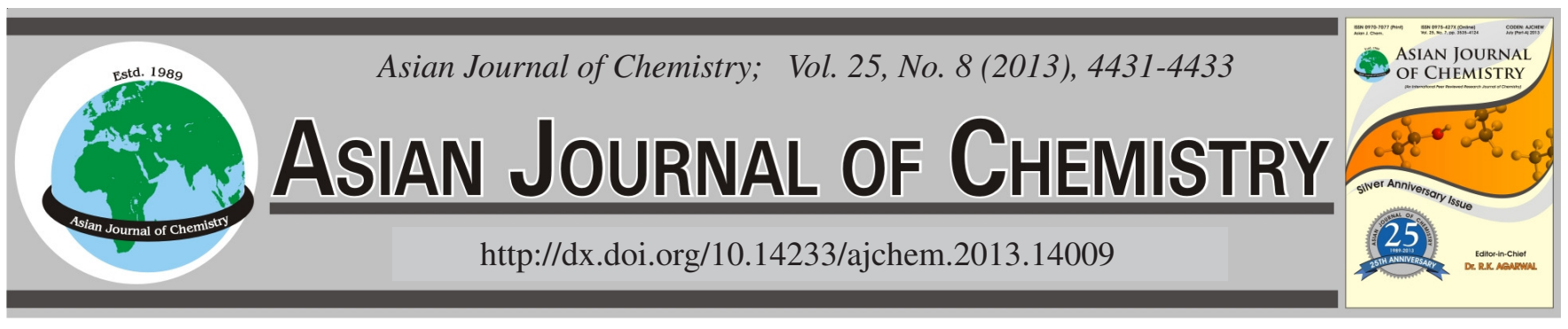

\title{
Removal of Humic Acid from Aqueous Solution by Dimethyl Diallyl Ammonium Chloride and Acrylamide
}

\author{
Peng Zhang*, Bozhi Ren, Yi Zhou and Kejia Liu
}

College of Cival Engineering, Hunan University of Science and Technology, Xiangtan 411201, Hunan Province, P.R. China

*Corresponding author: Fax: +86 731 58290052; Tel: +86 731 58290052; E-mail: zhangpeng388@126.com

The removal of organic pollutant humic acid from aqueous solution by flocculants were investigated. Two kinds of inorganic flocculants polyaluminium chloride, polymeric ferric sulfate and two kinds of organic flocculants polydimethyl diallyl ammonium chloride, dimethyl diallyl ammonium chloride and acrylamide (PDA) were used, the PDA had the best effect. Then the single influential factor such as: the dosage of the flocculants, the stirring time at the second stage, the $\mathrm{pH}$ value and the initial concentration of the humic acid solution were investigated. When the initial concentration was $10.0 \mathrm{mg} / \mathrm{L}$, the $\mathrm{pH}$ value was 5.0 and the dosage of PDA was $0.2 \mathrm{mg} / \mathrm{L}$, the removal rate could reach the maximum $94.6 \%$.

Key Words: Removal, Humic acid, Aqueous solution, Flocculants.

\section{INTRODUCTION}

Humic acid is a kind of nature organic matter which widely exits in the nature water body $y^{1,2}$. It is a class of organic substances which through a series of chemical reactions to generate from the remains of plants and animals. In the molecule structure of the humic acid the existence of phenolic groups and carboxyl causes the molecule take negative charges in aqueous solutions ${ }^{3}$. Humic substances according to their solubility in acid and alkali can be divided into the following three fractions e.g., the first is easily precipitated in acid soluble in alkaline liquids, known as humic acid. The second is both difficult to dissolve in acid, insoluble in alkali, known as humin and the third is not only soluble in acid and dissolved in alkali, known as fulvic acid ${ }^{4,5}$. Humic acid widely exists in the source water and the concentration of this organic matter in natural drinking water usually at $10 \mathrm{mg} / \mathrm{L}$ and the percentage of the humic acid is $50-90 \%$ for the total organic matter. Humic substances can react with chlorine during drinking water treatment and produce disinfection byproducts (DBPs), such as trihalomethanes with potential adverse health impacts ${ }^{6}$. The presence of humic substances in water introduces a yellowish to brown colour. Moreover, high affinity of humic substances for complexation with various pollutants including heavy metals and pesticides causes contamination of ground and surface water ${ }^{7-10}$. Therefore, the presence of humic acid in water resource is an urgent problem.

Several techniques have been developed in the field of water treatment, such as: flocculation, sedimentation, advanced oxidation processes (AOPs) and gravity filtration ${ }^{11}$. Flocculation is one kind of essential and the cheapest process for the water treatment and widely used ${ }^{12}$. Polymerization of diallyl dimethyl ammonium chloride and acrylamide (PDA) is one of the typical kind organic flocculants and has become most widely used in the field of oil exploration, paper making, textile printing, industrial wastewater treatment and sludge dewatering, etc. ${ }^{13,14}$.

In the present study, PDA was used for the removal of humic acid from aqueous solutions. Firstly, the common flocculants such as PDA, PDMDAAC, PFS and PAC used, when using the flocculants PDA, it could achieve the great removal rate and under the lowest dosage. The effects of variables such as dosage, stirring time, $\mathrm{pH}$ value and the initial concentration of humic acid were studied and achieved satisfied result.

\section{EXPERIMENTAL}

Industrial grade humic acid was purchased from Sinopharm Chemical Reagent Co. Ltd.; industrial grade polydimethyl diallyl ammonium chloride (PDMDAAC) was purchased from Chongqing Blue Clean Water Material Co. Ltd.; polymerization of dimethyl diallyl ammonium chloride and acrylamide (PDA) was laboratory-made.

The instruments used in the experimental setup were as follows: ZR4-6 Jar Tester supplied by Zhongrun Water Industry Technology Development Co. Ltd. from Shenzhen, China; 
UV-VIS spectrophotometer (TU-1910) supplied by Beijing Purkinje General Instrument Co. Ltd.

Wastewater sample: Take $1 \mathrm{~g}$ humic acid with the electronic balance, dissolved in $250 \mathrm{~mL}$ beaker and added some $1 \mathrm{~mol} / \mathrm{L} \mathrm{NaOH}$ solution in order to enhance the solubility of humic acid, then transferred the solution into a $1000 \mathrm{~mL}$ volumetric flask. The flask was filled up to $1000 \mathrm{~mL}$ with distilled water to reached $1000 \mathrm{mg} / \mathrm{L}$. Then placed in the refrigerator to save. The $\mathrm{pH}$ of humic acid solution was 6.2 and the UV254 of humic acid solution was $0.285 \mathrm{~cm}^{-1}$, when doing the flocculation test, diluted it to $10 \mathrm{mg} / \mathrm{L}$ and the $\mathrm{pH}$ of solution was 8 .

Flocculation test: A ZR 4-6 stirring machine (Shenzhen Zhongran Water Industry Technology Development Co. Ltd., Shenzhen, China) with six stirrers was used in this experiment. $500 \mathrm{~mL}$ of wastewater was transferred into a beaker. Flocculants were dosed under medium stirring speed of $300 \mathrm{rpm}$ for $2 \mathrm{~min}$ and then changed to the speed of $70 \mathrm{rpm}$ for $10 \mathrm{~min}$. After, quiescent settling of $30 \mathrm{~min}$, samples were collected from $2 \mathrm{~cm}$ below the surface for measurement of $\mathrm{UV}_{254}$ using UV-VIS. Translated into concentration to calculate the removal rate.

\section{RESULTS AND DISCUSSION}

\section{Single influential factor analysis}

Effect of the dosage on humic acid removal rate: In this experiment, the effect of flocculants dosage on the humic acid removal efficiency was investigated with an initial humic acid concentration at $10.0 \mathrm{mg} / \mathrm{L}$ and the dosage of PDA and PDMDAAC range from $0.1 \mathrm{mg} / \mathrm{L}$ to $0.5 \mathrm{mg} / \mathrm{L}$, the dosage of PFS and PAC range from $7.0 \mathrm{mg} / \mathrm{L}$ to $35.0 \mathrm{mg} / \mathrm{L}$, not adjust the $\mathrm{pH}$ value.

Fig. 1 shows that the removal efficiency of humic acid by organic flocculants had the better removal efficiency than inorganic flocculants. PDA changed from a low value of $59.1 \%$ at $0.1 \mathrm{mg} / \mathrm{L}$ to its maximum of $90.1 \%$ at $0.2 \mathrm{mg} / \mathrm{L}$ and then decreased to $49.8 \%$ at the dosage of $0.5 \mathrm{mg} / \mathrm{L}$. The removal efficiency was increased from $34.1 \%$ at $0.1 \mathrm{mg} / \mathrm{L}$ to $50.9 \%$ at $0.5 \mathrm{mg} / \mathrm{L}$ as the increased of PDMDAAC dosage. And the two inorganic flocculants had the similar removal trend, the removal efficiency increased with the increased of PFS dosage and the removal efficiency reached $76.3 \%$ at the dosage of $21.0 \mathrm{mg} / \mathrm{L}$, at the same time, the removal efficiency increased with the increased of PAC dosage and the maximum was $70.5 \%$ at the dosage of $28.0 \mathrm{mg} / \mathrm{L}$. As the dosage further increased, the removal efficiency decreased. The reason was that when flocculants was over dosage, the positive charge of flocs increased, furthermore, repulsion interaction among flocs was enhanced and so flocs were restabilized and dispersed in treated samples.

Effect of stirring time on humic acid removal rate: In this experiment, the effect of stirring time at the second stage on the humic acid removal efficiency was investigated with an initial humic acid concentration of $10 \mathrm{mg} / \mathrm{L}$ and the stirring time range from $4 \mathrm{~min}$ to $12 \mathrm{~min}$, not adjust the $\mathrm{pH}$ value (Fig. 2).

The determination of optimum stirring time for humic acid removal rate was carried out at 4-12 min. It was known that stirring time influenced the removal rate, when the stirring time was $10 \mathrm{~min}$ at the second stage, the removal rate could reach its maximum at $92.7 \%$. When treating humic acid wastewater, PDA flocculants showed both charge neutralization and adsorption bridging functions. When the stirring time was too short, it made the flocculants and the humic acid molecules in insufficient contact with each other and could not play the charge neutralization and adsorption bridging functions very well. But when the stirring time was too long, it could decrease the adsorption bridging functions of PDA flocculants and the absorbed small flocs of the humic acid molecule was broken, which lead the humic acid molecules in flocs released into the water again, so the removal efficiency decreased.
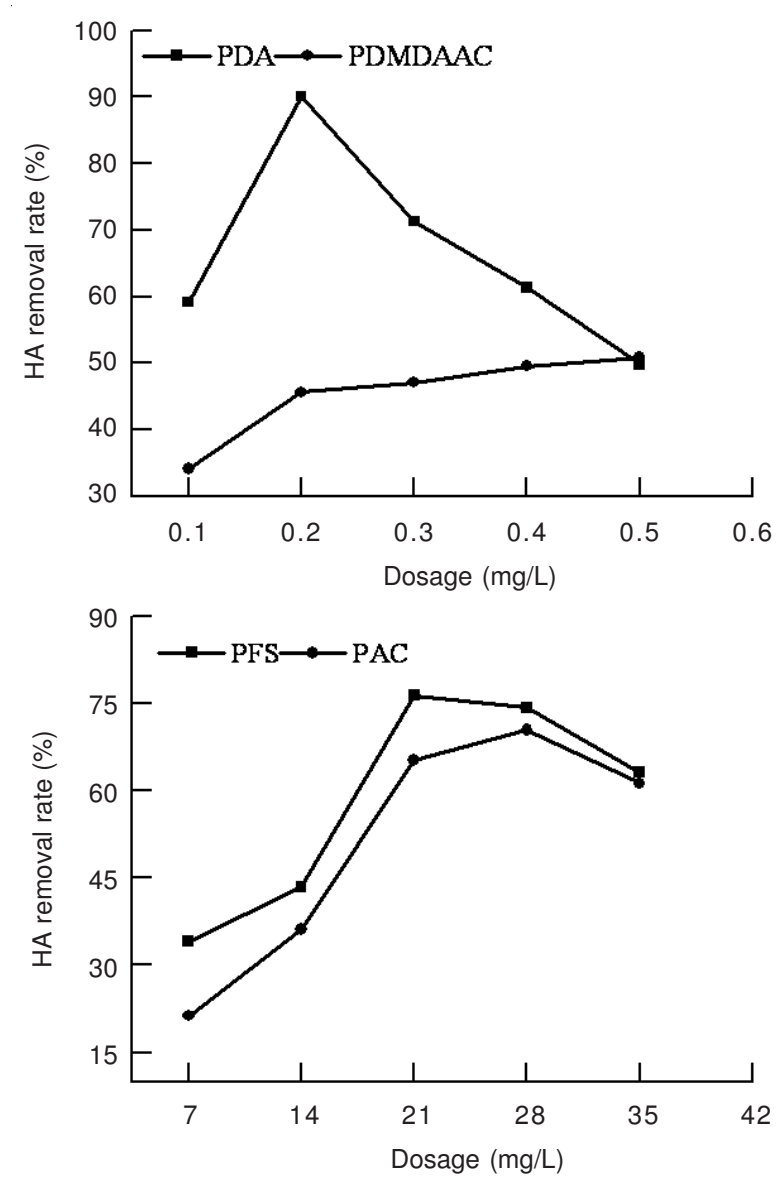

Fig. 1. Effect of flocculants dosage on the removal rate of humic acid

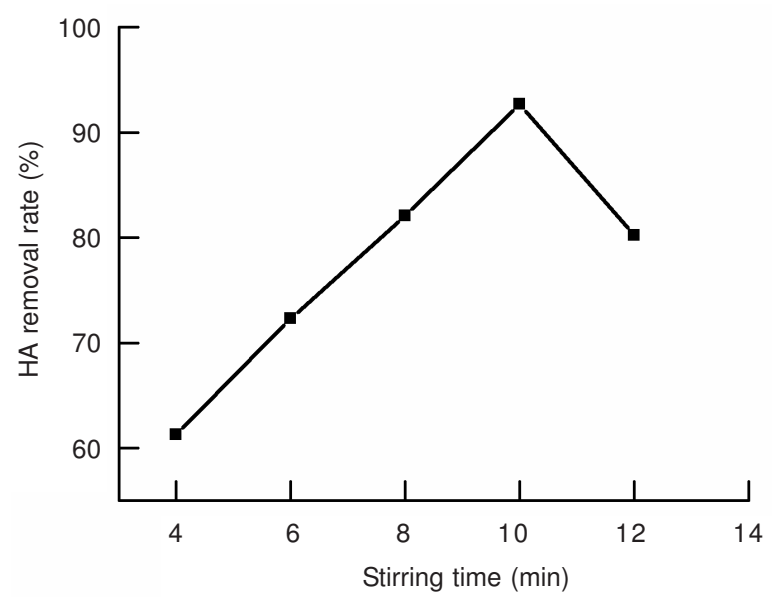

Fig. 2. Effect of stirring time on the removal rate of humic acid 
Effect of $\mathrm{pH}$ value on humic acid removal efficiency: In this experiment, the effect of $\mathrm{pH}$ value on the removal efficiency of humic acid was investigated with an initial humic acid concentration of $10 \mathrm{mg} / \mathrm{L}$, the dosage of PDA was 0.2 $\mathrm{mg} / \mathrm{L}$ and the $\mathrm{pH}$ range from 5 to 9 (Fig. 3).

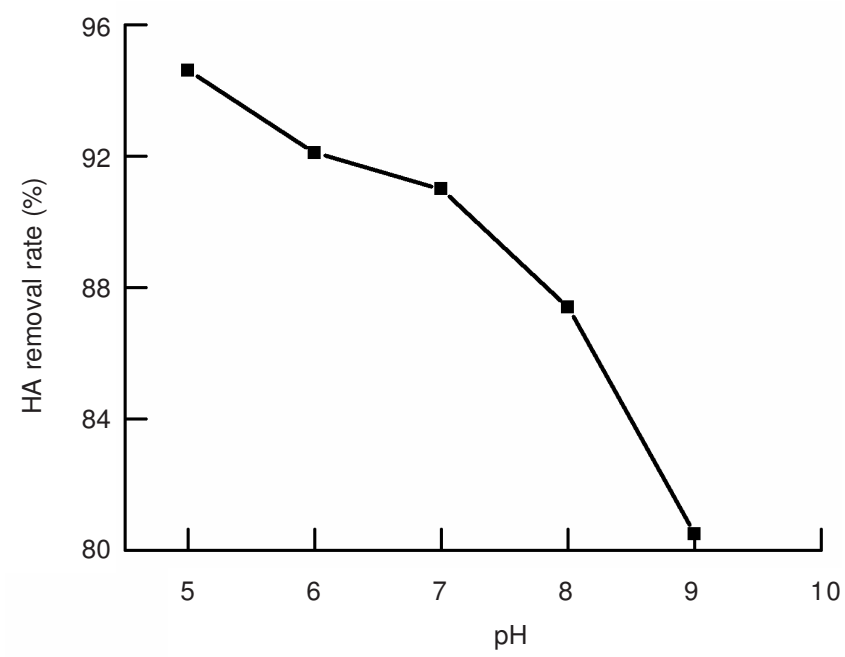

Fig. 3 Effect of $\mathrm{pH}$ on the removal rate of humic acid

The $\mathrm{pH}$ value is an important factor affecting the flocculation. On increasing of the $\mathrm{pH}$ value, the humic acid removal rate decreased, under the weak acidic conditions. When the $\mathrm{pH}$ value was 5 , the flocculants had the best effect and the removal rate could reached $94.6 \%$. Under the low $\mathrm{pH}$ value, the solution had the positive charges and the humic acid had the negative charges, so the charge neutralization lead the humic acid molecules and the flocculants molecules attracted each other very well, so the removal rate was increased as the decreased of the $\mathrm{pH}$ value. On the contrary, when the $\mathrm{pH}$ value higher, the solution existed a large number of $\mathrm{OH}^{-}$, so the positive charge of the PDA flocculants would attract with the $\mathrm{OH}^{-}$ which containing in the solution, which lead to the reduction of neutralization ability. So the removal rate decreased with the increase of the $\mathrm{pH}$ value.

Effect of initial concentration on removal efficiency of humic acid: In this experiment, the effect of initial concentration on the humic acid removal efficiency was investigated and the initial concentration range from $5 \mathrm{mg} / \mathrm{L}$ to $15 \mathrm{mg} / \mathrm{L}$, the dosage of PDA was $0.2 \mathrm{mg} / \mathrm{L}$, not adjust the $\mathrm{pH}$ value (Fig. 4).

The initial concentration of humic acid was an important parameter that determines the amount of removal rate. When fixed the dosage of the PDA flocculants, if the initial concentration of the humic acid changed, the removal rate would change and the removal rate reached the maximum at $89.3 \%$ at the initial concentration of humic acid was $12.5 \mathrm{mg} / \mathrm{L}$. At low initial concentration, $0.2 \mathrm{mg} / \mathrm{L}$ PDA flocculants had exceed the optimum dosage, so the redundant flocculants winded and covered with each other, which resulted in decrease of the removal rate. When the initial concentration was too high, the $0.2 \mathrm{mg} / \mathrm{L}$ PDA flocculants dosage have not reached the optimum value. So if the initial concentration not at same value and the optimum dosage of the flocculants would require.

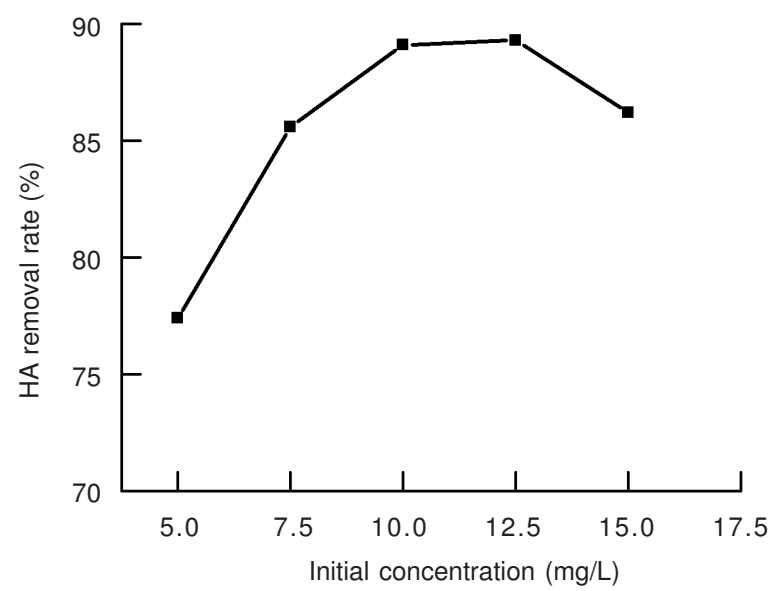

Fig. 4. Effect of initial concentration on the removal rate of humic acid

\section{Conclusion}

In this study, the single influential factor was investigated, including the kinds of the flocculants, the dosage of the flocculants, the stirring time at the second stage, the $\mathrm{pH}$ value and the initial concentration of the humic acid solution. When the humic acid initial concentration in aqueous solution was $10 \mathrm{mg} / \mathrm{L}$, using the PDA flocculants for removing the humic acid, the maximum removal rate could reach $94.6 \%$ when the $\mathrm{pH}$ value was 5.0 and the dosage of PDA was $0.2 \mathrm{mg} / \mathrm{L}$. The PDA flocculates in solution by charge neutralization and adsorption bridging mechanism.

\section{ACKNOWLEDGEMENTS}

The authors are grateful for the financial support provided by the National Natural Science Foundation of China (Project No.NSFC, 51174090) and the Education Department Key Project of Hunan Province (08A019).

\section{REFERENCES}

1. M.C. He, Y.H. Ye and C.Y. Lin, J. Environ. Sci., 20, 1294 (2008).

2. S. Bratskayaa, S. Schwarz and D. Chervonetsky, Water Res., 38, 2955 (2004).

3. W.S. Wan Ngah, M.A.K.M. Hanafiah and S.S. Yong, Colloids Surf. B., 65, 18 (2008)

4. A. Badis, F.Z. Ferradji, A. Boucherit, D. Fodil and H. Boutoumi, Desalination, 259, 216 (2010).

5. Y.H. Zhan, Z.L. Zhu, J.W. Lin, Y.L. Qiu and J.F. Zhao, J. Environ. Sci., 22, 1327 (2010).

6. L. Zhao, F. Luo, J.M. Wasikiewicz, H. Mitomo, N. Nagasawa, T. Yagi, M. Tamada and F. Yoshii, Bioresour. Technol., 99, 1911 (2008).

7. A. Imyim and E. Prapalimrungsi, J. Hazard. Mater., 184, 775 (2010).

8. L.T. Shirshova, E.A. Ghabbour and G. Davies, Geoderma, 133, 204 (2006).

9. J. Liu, J. Wang, Y.H. Chen and H. Lippmann-Pipke, J. Environ. Sci., 22, 1695 (2010).

10. Q.Y. Yue, Y. Li and B.Y. Gao, Colloids Surf. B., 72, 241 (2009).

11. X. Zhan, B.Y. Gao, Q.Y. Yue, B. Liu, X. Xu and Q. Li, J. Hazard. Mater., 183, 279 (2010).

12. H.L. Zheng, X.P. Sun, Q. He, K. Liang and P. Zhang, J. Appl. Polym. Sci., 110, 2461 (2008).

13. A.T. Horvath, A.E. Horvath, T. Lindstrom and L. Wagberg, Langmuir, 24, 7857 (2008).

14. R. Losada and C. Wandrey, Macromolecules, 42, 3285 (2009). 